\title{
Staged Hand Salvage and Reconstruction with Three Free Tissue Transfers: A Ten-year Follow-up
}

\author{
Steffen U. Eisenhardt, $\mathrm{MD}^{1 *}$; Jurij Kiefer, $\mathrm{MD}^{\mathbf{1}}$; Horst Zajonc, $\mathrm{MD}^{\mathbf{1}}$; Arash Momeni, $\mathrm{MD}^{2}$; Gerhard Björn \\ Stark, MD ${ }^{1}$
}

${ }^{1}$ Department of Plastic and Hand Surgery, University of Freiburg Medical Center, Freiburg, Germany

${ }^{2}$ Division of Plastic Surgery, Stanford University Medical Center, Palo Alto, USA

\begin{abstract}
A 27-year-old male suffered a severe work-related crush injury to his left dominant hand, resulting in a composite defect, including necrosis of the thumb and fingers as well as soft tissues distal to the radiocarpal joint. An amputation of the affected fingers had to be performed at the level of the metacarpophalangeal joints. In an attempt to prevent necrosis of the exposed carpal bones and metacarpal stumps, immediate coverage was achieved by reconstruction with a free rectus abdominis muscle flap, two weeks after the initial trauma. After six months, thumb reconstruction was accomplished by second toe transfer from the right foot. After another four months, the contralateral second toe was utilized for reconstruction of the fifth digit. Within two years after the accident, the patient returned to full-time occupational activities. He demonstrated excellent motor function, satisfactory discriminatory sensation to the transferred toes as well as protective sensation to the skin graft covering the flap. The donor site morbidity of both the toes and the rectus abdominis muscle was acceptable. After ten years following reconstruction, the patient had fully adjusted to his disability and was in full-time occupational activity with only minor limitations in his everyday life.
\end{abstract}

\section{Introduction}

Free tissue transfers, including composite tissues, e.g., toes, have become a routine procedure in reconstructive hand surgery. However, consecutive microsurgical transplants to the same defect area, particularly to the hand, with subsequent long-term follow-up, have rarely been reported. For complete loss of hand function, the alternatives are limited. Prostheses have the drawback of lacking sensational feedback, and hand transplantation may be justified in the setting of bilateral amputees to avoid unnecessary immunosuppression [1]. Thus, any attempt to preserve all viable tissues by early coverage with vascularized tissues is mandatory. Ideally, the chosen procedure should have several characteristics for immediate reconstruction, i.e., be technically simple, safe and allow for coverage of large soft tissue defects.

Free toe transfers for thumb and finger reconstruction are established techniques. However, adequate preoperative planning with consideration as to the choice of the donor, either first or second toe or an en-bloc transfer of two toes, has to be carried out. Advantages and pitfalls of the respective procedures with regard to safety and donor site morbidity have to be considered.

Here, we describe a case of an early staged reconstruction by which the exposed carpal and metacarpal bones were salvaged by a free rectus muscle transfer, followed by two second toe transfers with a functional follow-up after ten years.

*Correspondence: Steffen Ulrich Eisenhardt, MD

Department of Plastic and Hand Surgery, University of Freiburg Medical Center, Freiburg, Germany. E-mail: steffen.eisenhardt@uniklinik-freiburg.de

Received: Apr. 14, 2017; Accepted: Sep. 11, 2017; Published: Oct. 11, 2017

International Microsurgery Journal. 2017;1(2):3

DOI: $10,24983 /$ scitemed.imj.2017.00037

Copyright ( $\subset 2017$ The Author(s). This is an open-access article distributed under the terms of the Creative Commons Attribution 4.0 International License (CC-BY).

\section{Case Report}

A 27-year-old manual worker suffered a severe occupational crush injury to the entire left dominant hand (Figure 1A). In detail, the volar aspect of the affected hand was completely degloved with an avulsion injury to the arteries distal to the proximal interphalangeal joints. The volar arch was intact, and the volar arteries were in continuation, but severely elongated. Furthermore, the work-related accident caused a complete avulsion of the thumb and large soft tissue defect of the fifth digit. At the time of the initial presentation in our unit, the perfusion of all fingers seemed to be sufficient, and, thus, no attempt was made at this stage to reconstruct the arteries by using vein grafts. Consequently, the soft tissue defects were carefully debrided and readapted. However, after several days, the perfusion of all fingers proved to be insufficient, which ultimately led to necrosis of the fingers including the soft tissue coverage of the hand distal to the radiocarpal joint (Figure 1B).

After complete demarcation of the necrotic tissue, we performed a radical necrectomy of all non-vital tissues, including the amputation of the thumb and fingers. Immediate vascularized coverage to salvage the remnant exposed carpal and metacarpal bones was achieved by transferring of the right rectus abdominis muscle as a free flap. The microsurgical reconstruction was established with an end-to-side anastomosis of the deep inferior epigastric vessels to the radial artery and an end-to-end anastomosis to the cephalic vein, respectively. Subsequently, the free muscle transfer was covered by split-thickness skin grafting (Figure 1C). The postoperative recovery was uneventful. The skin graft and flap finally became thin and pliable due to regression of the initial edema and neurogenic atrophy. Furthermore, the patient did not develop any abdominal herniation due to harvesting of the rectus abdominis muscle.

After six months, a second toe transfer from the right foot to the first metacarpal ray was performed to reconstruct the thumb. An anatomic variation of the first dorsal metatarsal artery complicated the harvest of the toe. The dorsalis pedis artery was rudimentary and ended just distal to the extensor retinaculum. Thus, the very short plantar vascular pedicles supplied by 


\section{Case Report}
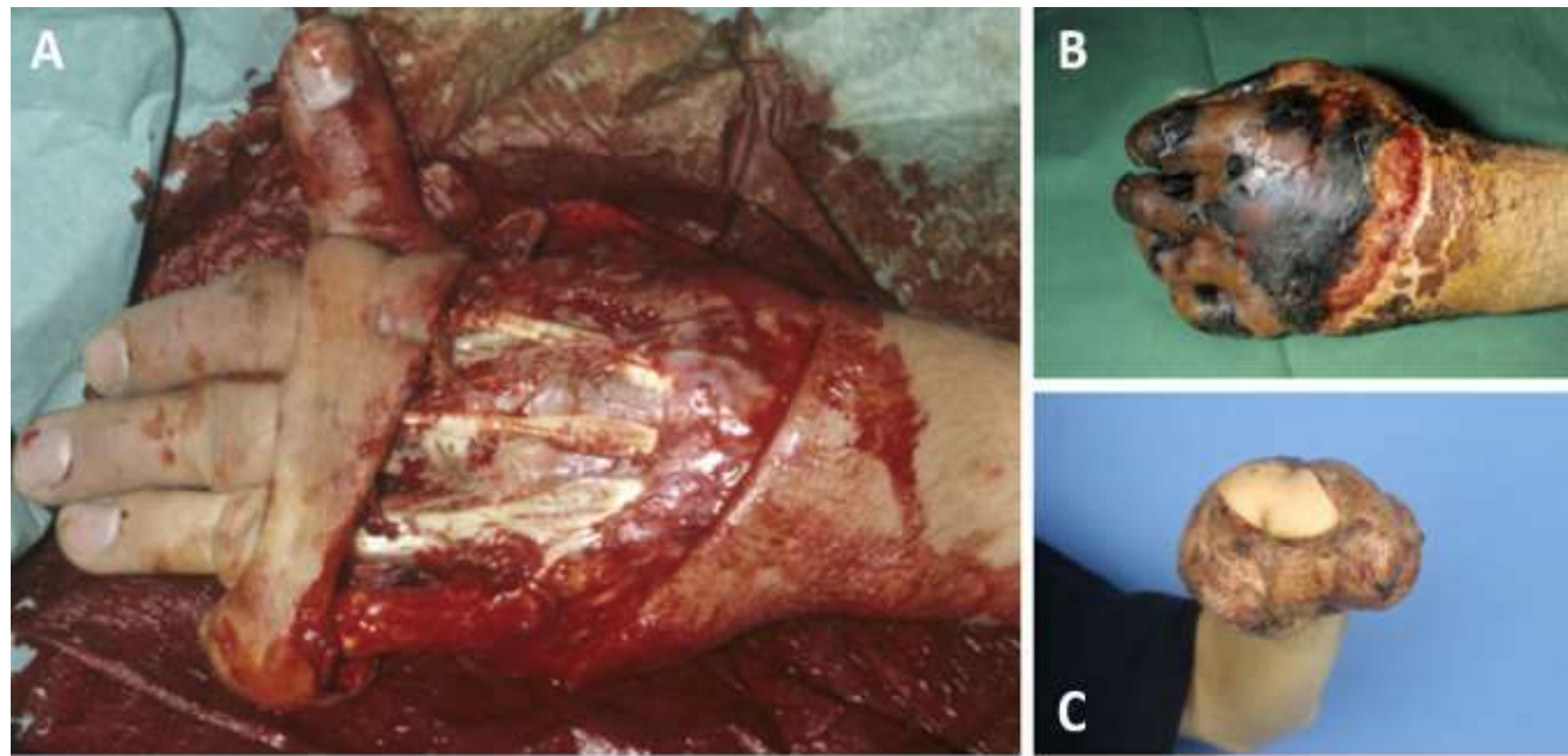

Figure 1. A, severe crush injury to the left dominant hand of a 27-year-old patient causing a complete degloving distal to the radiocarpal joint. B, subsequent necrosis of the thumb and fingers. C, postoperative result after amputation of the thumb and fingers, and stable defect coverage with a split-thickness skin grafted myocutaneous rectus abdominis free flap.

the medial plantar artery had to be dissected via a dorsal approach established between the first and second metatarsal bones. Microsurgical anastomosis was accomplished between the inferior epigastric artery of the previously transferred rectus abdominis muscle and the first plantar metatarsal artery of the second toe in an end-to-side manner. Sufficient venous drainage
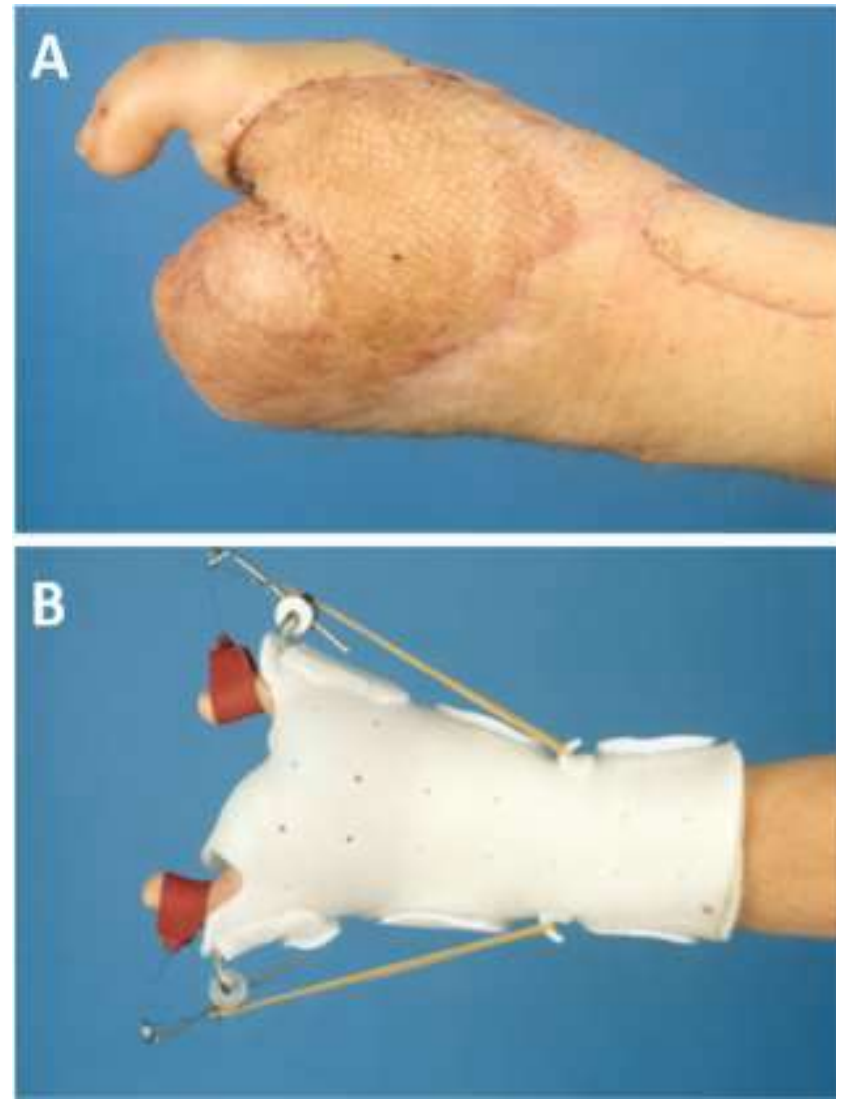

Figure 2. A, postoperative view after second toe transfer for thumb reconstruction. $\mathrm{B}$, postoperative physiotherapy regime after transplantation of the second toe. was ensured by two anastomoses between the subcutaneous veins of the toe and tributaries to the cephalic vein. Additionally, the plantar nerves of the toe were coapted to the former radial and ulnar nerves of the thumb. Care was taken to perform a stable wire-based osteosynthesis between the first metacarpal stump and the base of the proximal phalanx of the toe in a $30^{\circ}$ flexed angulation to compensate for the anatomic hyperextension of the toe (Figure $2 A)$. Active physiotherapy was initiated 14 days postoperatively.

After another four months, a standard second toe transfer from the left foot was performed to reconstruct the fifth ray. The harvesting of the toe revealed the same anatomic variation of the vessels described above for the right foot. In accordance with the thumb reconstruction, the arterial blood supply was established via microvascular end-to-side anastomosing of the first plantar metatarsal artery of the toe to the inferior epigastric artery of the rectus abdominis muscle. Venous drainage was ensured by two anastomoses between the subcutaneous veins of the toe and tributaries to the cephalic vein. Again, the microsurgical reconstruction was accompanied by an intensive physiotherapeutic regime postoperatively (Figure $2 \mathrm{~B}$ ). The wound healing process at all sites was uneventful.

The donor site morbidity of both the second toe transfers occurred to be relatively low. Shortly after the initial use of the orthopedic shoes, the patient could change to normal shoes. The patient did not complain about any longterm gait disorders or impairments in his everyday life. Within 21 months after the initial accident, the patient returned to his occupational activities. However, a change of his duties had to be arranged, resulting in the patient being full-time occupied as an industrial clerk.

Due to complaints involving the hypothenar and intermetacarpal areas of the fourth and fifth metacarpal bones, the patient returned to our clinic ten years after the initial reconstruction (Figure 3A). The patient complained pain, fatigue, and spasms of the intrinsic hand muscles; e.g., when driving a car for more than two hours. Psychologically, the patient adapted very well to the appearance of the hand and used his hand in everyday-life situations without any technical aids or attempts to cover the hand. Functionally, the patient achieved a good range of motion of the wrist with $40^{\circ}-0^{\circ}-40^{\circ}$ wrist extension/flexion, $15^{\circ}-0^{\circ}-25^{\circ}$ radial and ulnar abduction of the wrist, and free wrist pronation/supination. For the reconstructed thumb, a range of motion of $0^{\circ}-15^{\circ}-30^{\circ}$ extension/flexion of the metacarpal joint and $0^{\circ}-10^{\circ}-50^{\circ}$ extension/flexion of the proximal interphalangeal joint were documented. The distal interphalangeal joint of the reconstructed thumb only presented with a flexion of approximately $5^{\circ}$ and, therefore, was not measured specifically. For 


\section{Case Report}
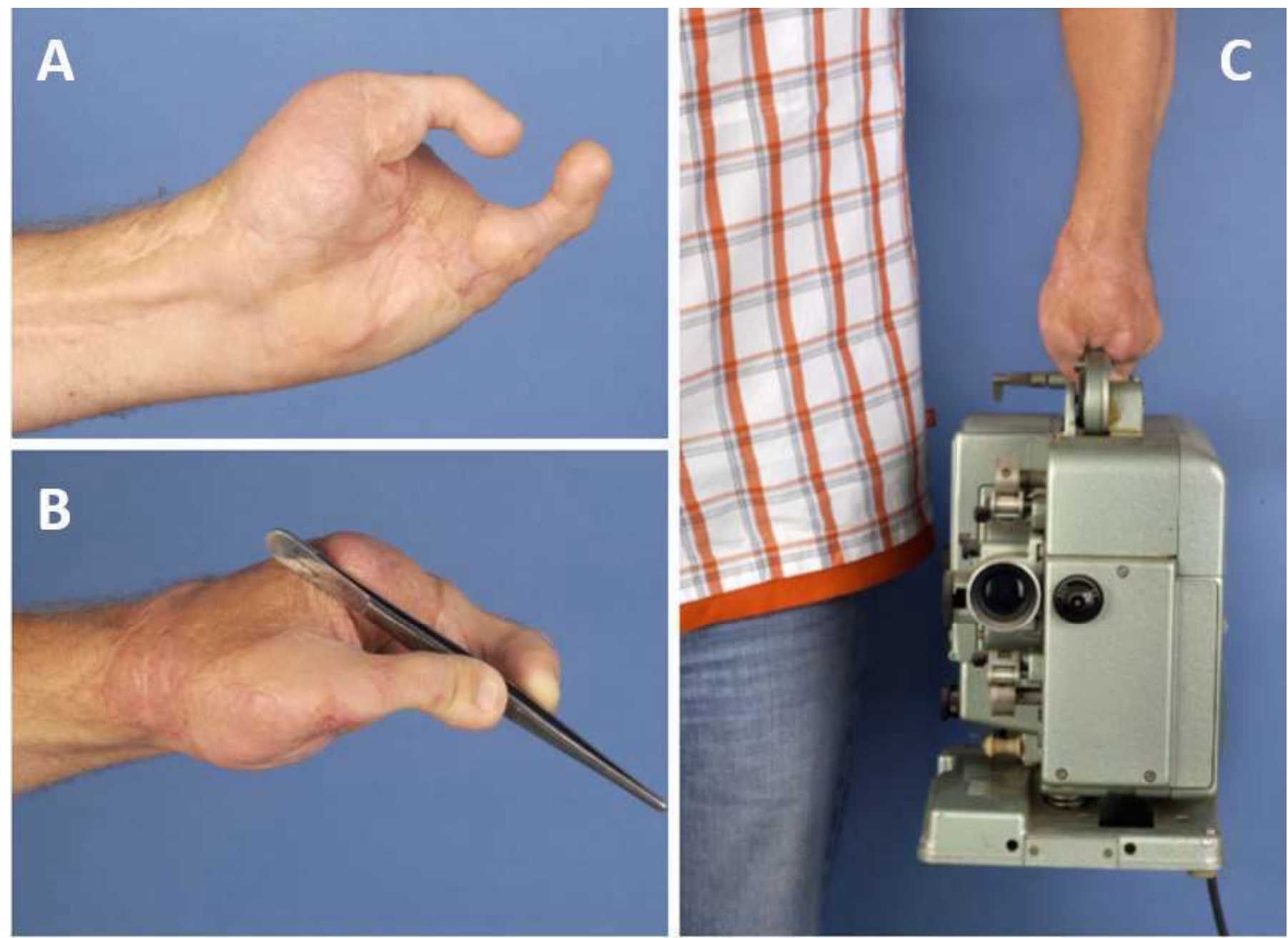

Figure 3. Long-term outcome, ten years after reconstruction. A, clinical appearance. B, stable pinch grip. C, powerful grip carrying up to five kg of weight.

the reconstructed ulnar finger, a range of motion of $0^{\circ}-0^{\circ}-30^{\circ}$ extension/flexion of the metacarpal joint and $15^{\circ}-15^{\circ}-70^{\circ}$ extension/flexion of the proximal interphalangeal joint was documented. The distal interphalangeal joint of this finger was documented with an active flexion of approximately $20^{\circ}$. Furthermore, the patient developed a good discriminatory sensation of the transferred toes and protective sensation in the flap area. In detail, the radial nerve of the reconstructed thumb revealed a 2-point discrimination of $10 \mathrm{~mm}$, whereas the ulnar nerve showed a 2-PD of $8 \mathrm{~mm}$. For the reconstructed ulnar digit, 2-PD measurements resulted in $10 \mathrm{~mm}$ for the radial nerve and protective sensation $(>10 \mathrm{~mm})$ for the ulnar nerve. The patient adapted well for motor use of the two reconstructed fingers for two-point fixation by direct opposition, as well as for three-point fixation (Figure 3B) and power grip (Figure $3 \mathrm{C}$ ) by using the stumps of the remaining metacarpal bones as a counter pole. The postoperative radiographs showed a stable bony fixation with appropriate angulation of the reconstructed digits.

Nevertheless, the patient has to make some effort to turn the reconstructed thumb into exact opposition due to missing thinner muscles and compensate this well with the ulnar intrinsic muscles, which may be the cause of the experienced pain when exercising a forced grip for more than two hours. Therefore, we recommended a pronational osteotomy of the thumb metacarpal of about $15^{\circ}$. However, the patient refused to undergo further surgery.

\section{Discussion}

Using the full armamentarium of reconstructive microsurgery allows a multitude of options for hand reconstruction. When distal skeleton still is preserved, any attempt should be made to salvage as much length and prevent infection by debridement and early, or even emergency vascularized coverage [2]. We generally prefer free vascularized tissue transfers over pedicled flaps like the pedicled groin or the pedicled contralateral lateral arm flap for complex hand reconstruction, as the use of pedicled flaps delays the postoperative physiotherapy regime. For large defects, as in this case, the use of a free rectus abdominis muscle flap covered with a split thickness skin graft is a valuable option, due to its size, technical ease, acceptable donor-site defect, and significant potential for subsequent thinning [3].

A muscle offers the best prerequisites to prevent infection [4] and, thus, we generally prefer it over perforator flaps like the thin anterolateral thigh flap [5] in the acute trauma situation. Another possible option for defects of similar size is the free gracilis muscle flap and free latissimus dorsi flap. In our experience, revisions or secondary procedures for thinning are usually not necessary with these pliable flaps, which even may develop some protective sensation. Clearly, perforator flaps are valuable options for complex hand reconstructions [6], but applying the above demonstrated reconstructive concept allows for performance of additional functional procedures. However, with the broad distribution and access to super-microsurgical techniques and instruments, severe degloving injuries similar to the demonstrated case are likely to be dealt more aggressively nowadays. Precisely, the perfusion of the fingers and soft tissue can be secured by replacing all affected arteries with vein grafts in the course of the initial surgical care.

Performing free microsurgical toe transfers for thumb reconstruction is undebatable [7], and the preference of the entire or tailored form of the great toe for this indication has been established for functional as well as aesthetic reasons [8]. With all fingers, including the thumb, being lost, considerations must be addressed to reconstruct at least two opposing rays. The harvest of the great toe and its concomitant donor site morbidity are not trivial with 


\section{Case Report}

regard to foot function [9]. The second toe transfer has less associated donor site morbidity, and the donor site can usually be closed primarily with an inconspicuous scar hidden within the web space dorsally and on the noweight-bearing area of the plantar foot. Another important pitfall for both reconstructive options is the anatomical variations in the origin of the first dorsal metatarsal (FDMA) artery $[10,11]$. In half of the patients, the FDMA arises directly from the dorsalis pedis artery or the superficial part of its deep perforating branch. The distal course of the FDMA in these patients is in a superficial plane. In $40 \%$ of the cases, the FDMA takes origin from the lower part of the deep communicating branch or the deep plantar arch. The artery runs within the first interosseous muscle and eventually becomes superficial between the head of the first and second metatarsal. In the remaining $10 \%$ of the patients, the FDMA is very narrow or completely absent. As demonstrated in our case, these patients' blood supply to the great and second toe largely depends on the first plantar metatarsal artery.

Furthermore, the willingness of a patient to accept a toe transfer has to be taken into consideration before performing such a procedure. In this context, aesthetic considerations may become secondary due to the goal of regaining grip function after the loss of the thumb and fingers, and, thus, a visible mutilation is unavoidable. Also, a thorough planning of the aspired grip function is necessary for a good match of the two poles. In the abovedescribed case, this goal was achieved by the bilateral use of two second toes. An alternative option for this case might have been a double digit transfer from the same foot [12] or a great toe transfer for thumb reconstruction combined with an en-bloc-transfer of the second and third toes [13]. However the concomitant donor site morbidity with these reconstructive procedures is not deniable. The risk for permanent gait disorders is higher with multiple toes sacrificed from the same foot. This is particularly the case for thumb reconstructions using the great toe flap because the great toe is mainly responsible for the push-off phase of gait. Thus, sacrificing the second toe only ensures minimal disruption to the gait function. In addition, achieving stable bony fixation of the transplanted toes in a sufficient palmar angulation is crucial to compensate for the anatomic hyperextension, as seen in our case.

\section{Conclusion}

Health care systems in many countries are confronted with increasing economic limitations. Thus, complex microsurgical procedures and extensive rehabilitation programs are poorly compensated. However, this case demonstrates a dramatic reduction of socioeconomic expenses by allowing a potential radiocarpal amputee to return to work for another estimated 30 years.

\section{Keywords}

Toe to hand transfer; emergency free flap; hand reconstruction.

\section{Article Information}

\section{Conflict of Interest Disclosures: None}

Funding: None

\section{References}

1. Brandacher $G$, Ninkovic $M$, Piza-Katzer $H$, et al. The Innsbruck hand transplant program: update at 8 years after the first transplant. Transplant Proceedings 2009; 41: 491-494.

2. Horch RE, Walgenbach KJ, Voigt M, Stark GB. [The free "emergency" rectus abdominis flap transfer for coverage of complex hand injuries]. Langenbecks Archiv fur Chirurgie. Supplement. Kongressband. Deutsche Gesellschaft fur Chirurgie. Kongress. 1998; 115: 1194-1196.

3. Horch RE, Stark GB. The rectus abdominis free flap as an emergency procedure in extensive upper extremity soft-tissue defects. Plastic and Reconstructive Surgery 1999; 103: 1421-1427.

4. May JW Jr., Gallico GG 3rd, Lukash FN. Microvascular transfer of free tissue for closure of bone wounds of the distal lower extremity. The New England Journal of Medicine 1982; 306: 253-257.

5. Adani R, Tarallo L, Marcoccio I, Cipriani R, Gelati C, Innocenti M. Hand reconstruction using the thin anterolateral thigh flap. Plastic and Reconstructive Surgery 2005; 116: 467-473.

6. Kim YH, Ng SW, Youn SK, Kim CY, Kim JT. Use of latissimus dorsi perforator flap to facilitate simultaneous great toe-to-thumb transfer in hand salvage. Journal of Plastic, Reconstructive \& Aesthetic Surgery: JPRAS 2011; 64: 827-830.

7. Buncke $\mathrm{HJ}$, Buncke GM, Lineaweaver WC, et al. The contributions of microvascular surgery to emergency hand surgery. World Journal of Surgery 1991; 15: 418-428.

8. Foucher G, Nagle DJ. Microsurgical reconstruction of fingers and fingertips. Hand Clinics. 1999; 15: 597-606.

9. Michon J, Merle M, Bouchon Y, Foucher G. Thumb reconstruction pollicisation or toe-to-hand transfers. A comparative study of functional results. Annales de Chirurgie de la main: organe officiel des societies de Chirurgie de la main 1985; 4: 98-110.

10. Koshima I, Etoh H, Moriguchi T, Soeda S. Sixty cases of partial or total toe transfer for repair of finger losses. Plastic and Reconstructive Surgery 1992; 92: 1331-1338.

11. Lutz BS, Wei FC. Basic principles on toe-to-hand transplantation. Chang Gung Medical Journal. 2002; 25: 568-576.

12. Galpern DW, Tsai TM. Multiple toe transfer and sensory free flap use after a traumatic amputation of multiple digits. Surgery done in a single setting: a case study. Microsurgery 2011; 31: 484-489.

13. Buncke GM, Buncke HJ, Oliva A, Lineaweaver WC, Siko PP. Hand reconstruction with partial toe and multiple toe transplants. Clinics in Plastic Surgery. 1992; 19: 859-870. 